\title{
Important preparatory steps and clinical considerations for pharmacogenetics adoption into practice
}

\author{
Aniwaa Owusu Obeng ${ }^{1,2,3}$, Nihal El Rouby ${ }^{4}$, Michelle Liư ${ }^{5}$ Rick Wallsten ${ }^{6}$ \\ 1The Charles Bronfman Institute for Personalized Medicine, Icahn School of Medicine at Mount Sinai, New York, NY 10029, USA. \\ 2Department of Genetics and Genomic Sciences, Icahn School of Medicine at Mount Sinai, New York, NY 10029, USA. \\ ${ }^{3}$ Pharmacy Department, The Mount Sinai Hospital, New York, NY 10029, USA. \\ ${ }^{4}$ Department of Pharmacy Practice and Administrative Services, James Winkle College of Pharmacy, University of Cincinnati, \\ Cincinnati, OH 45229, USA. \\ ${ }^{5}$ Department of Pharmacy, Vanderbilt University Medical Center, Nashville, TN 37212, USA. \\ ${ }^{6}$ Sema4, Stamford, CT 06902, USA.
}

Correspondence to: Dr. Aniwaa Owusu Obeng, The Charles Bronfman Institute for Personalized Medicine, Icahn School of Medicine at Mount Sinai, One Gustave L. Levy Place, Box 1003, New York, NY 10029, USA.

E-mail: aniwaa.owusu-obeng@mssm.edu

How to cite this article: Owusu Obeng A, El Rouby N, Liu M, Wallsten R. Important preparatory steps and clinical considerations for pharmacogenetics adoption into practice. J Transl Genet Genom 2021;5:64-79.

http://dx.doi.org/10.20517/jtgg.2020.52

Received: 18 Nov 2020 First Decision: 23 Nov 2020 Revised: 1 Dec 2020 Accepted: 9 Dec 2020 Available online: 2 Jan 2021

Academic Editor: Ramón Cacabelos Copy Editor: Miao Zhang Production Editor: Jing Yu

\begin{abstract}
Genetic influence on medication response has been well documented; yet, the clinical integration of pharmacogenetics has been slow. Lack of knowledge on the fundamentals of pharmacogenetic testing among clinicians and the complexities surrounding test selection, results interpretation, and clinical utility, among others, contribute to this slow adoption. In this paper, we describe integration of pharmacogenetics in the clinic, selection of target population and testing laboratories, team of expertise needed, available resources, and critical considerations for accurate interpretation of test results. We also discuss phenoconversion and pharmacogenetic utility in special populations including pediatrics, pregnant women and transplant patients. Ultimately, it should be observed that pharmacogenetics is an additional piece in the prescribers' toolbox to guide prescribing decisions. Other intrinsic and extrinsic factors should be considered for more accurate and personalized prescribing practice. This paper aims to guide clinicians with preparatory steps and "clinical pearls" necessary to successfully integrate pharmacogenetics into routine practice.
\end{abstract}

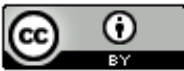

(C) The Author(s) 2021. Open Access This article is licensed under a Creative Commons Attribution 4.0 International License (https://creativecommons.org/licenses/by/4.0/), which permits unrestricted use, sharing, adaptation, distribution and reproduction in any medium or format, for any purpose, even commercially, as long as you give appropriate credit to the original author(s) and the source, provide a link to the Creative Commons license, and indicate if changes were made. 
Keywords: Pharmacogenomics, pharmacogenetic testing, personalized medicine, precision medicine, phenoconversion, pharmacogenetics in special populations, pharmacogenetics

\section{INTRODUCTION}

Medical professionals have long observed that some individuals respond differently to treatments than expected. Standard therapeutic doses have been proven to be ineffective and even harmful for some individuals ${ }^{[1,2]}$. Such interpatient variability can be attributed to a wide host of factors, including the genetic makeup of the individual ${ }^{[3]}$. Pharmacogenetics - the study of how genes influence one's response to medications - is an application of personalized medicine with clinically actionable examples ${ }^{[4]}$. Elements necessary for clinical implementation of pharmacogenetics such as robust and consistently replicated scientific evidence, affordable and high-throughput testing technologies, electronic health records (EHR)-enabled clinical decision support (CDS), and expert-driven resources for pharmacogeneticsguided therapeutic recommendations, are all available and in use at some academic and non-academic institutions ${ }^{[4,5]}$. However, its widespread adoption is still lagging behind. Efforts from Pharmacogenomic Knowledgebase (PharmGKB) and professional societies such as the Clinical Pharmacogenetic Implementation Consortium (CPIC) and the Dutch Pharmacogenetic Working Group (DPWG) are actively ongoing and helping to overcome the challenge related to evidence and clinical guidance ${ }^{[4,6]}$. Early adopter and implementation programs in collaboration with federal networks/consortia are also working to address the remaining challenges and provide process best-practices for this discipline ${ }^{[7]}$. For instance, patient education materials and protocol resources have been developed by the Clinical Sequencing Evidence-Generating Research (CSER) (https://cser-consortium.org/). The Electronic Medical Records and Genomics (eMERGE) Consortium investigators developed validated electronic phenotyping algorithms named Phenotype Knowledge Base (https://phekb.org/) and also curated member-sites' implemented clinical decision support rules on their Clinical Decision Support Knowledge Base (https://cdskb.org/). In addition, the Implementing Genomics in Practice (IGNITE) Network has developed the Spark Toolbox (https://gmkb.org/ignite-toolbox-overview/) which hosts educational and implementation resources for researchers and clinicians.

Most clinicians agree that genetics impact response to medications ${ }^{[8]}$, and are favorable towards pharmacogenetics in general $^{[9]}$; however, their lack of appreciable knowledge of pharmacogenetic utility in practice have led to some resistance and slow uptake ${ }^{[9]}$.

The purpose of this manuscript is to provide stakeholders, particularly clinicians, who are interested in pharmacogenetic implementation with pre-implementation preparatory steps and other considerations for effective clinical adoption. We also discuss available pharmacogenetic resources, how to select genes and laboratories for testing and how to interpret and utilize the results to guide prescribing decisions [Table 1]. This manuscript is intended to provide a broad overview of pertinent points to consider prior to embarking on integration of this discipline into routine practice. Several actionable pharmacogenetic drug-gene pairs associated with psychiatry, pain, cardiology, and others are highlighted throughout the paper as examples for the various discussion points.

\section{SELECTING THE MEDICATIONS, GENES, AND THE LABORATORY FOR TESTING}

\section{The medications}

As of August 2020, there are 24 CPIC guidelines covering approximately 46 medications from cardiology to pain, infectious diseases, oncology, psychiatry, and others. A full list of the guidelines can be accessed via the CPIC website (https://cpicpgx.org/guidelines/). The United States Food and Drug administration (FDA) has also included pharmacogenetic information in the drug labeling for $279 \operatorname{drugg}^{[10]}$. This information is 
Table 1. Key preparatory steps in establishing pharmacogenetic implementation programs

\begin{tabular}{|c|c|c|}
\hline Preparatory steps & Elements & Points to consider \\
\hline \multirow[t]{3}{*}{$\begin{array}{l}\text { Selecting the } \\
\text { medications, genes and } \\
\text { the laboratory }\end{array}$} & The medications & $\begin{array}{l}\text { - Resources available include CPIC, FDA Table for Pharmacogenomic Associations, } \\
\text { DPWG Guidelines, FDA Tables of Pharmacogenetic Biomarkers, etc. } \\
\text { - Clinically actionable examples can be found in many clinical specialties such } \\
\text { as cardiology, pain, psychiatry, infectious diseases, oncology, gastroenterology, } \\
\text { transplantation, neurology, and others } \\
\text { - Some high-risk PGx medications are backed by randomized controlled trials, e.g., } \\
\text { - Abacavir, carbamazepine, and mercaptopurine } \\
\text { - Some high-risk PGx medications have black box warnings highlighting their PGx } \\
\text { associations, e.g., warfarin and clopidogrel }\end{array}$ \\
\hline & The genes & $\begin{array}{l}\text { - Gene selection is mainly driven by medications in a reactive testing model } \\
\text { - Of note, the } 24 \text { CPIC guidelines cover } 19 \text { genes } \\
\text { - Most early adopters initiate their programs with the CYP genes, e.g., CYP2C19, } \\
\text { CYP2D6, and CYP2C9 } \\
\text { - Other commonly implemented genes include VKORC1, SLCO1B1, and HLA-B } \\
\text { - Pre-emptive testing approach with a panel that covers common genes may reduce } \\
\text { the need to choose which gene(s) to test first } \\
\text { - Genes to be tested must have documented implications for medication response, } \\
\text { evidence of association with medication-related outcomes and pharmacogenetic- } \\
\text { guided therapeutic recommendation } \\
\text { - All relevant variants must be reviewed and interrogated during testing }\end{array}$ \\
\hline & The test and the lab & $\begin{array}{l}\text { - Laboratory must be CAP/CLIA accredited } \\
\text { - Test can be conducted in-house or with a commercia laboratory } \\
\text { - Commercial lab reports usually contains information on genes and variants tested, } \\
\text { genotypes, and phenotypes (pre-2019 reports may have included high-risk medications } \\
\text { and PGx-guided therapeutic recommendations) }\end{array}$ \\
\hline $\begin{array}{l}\text { Selecting the patients to } \\
\text { test }\end{array}$ & The patients & $\begin{array}{l}\text { - Consider patients who have experienced adverse drug events or failed therapies in } \\
\text { the past. } \\
\text { - Consider patients who are diagnosed with indications for which a PGx medication } \\
\text { treatment is anticipated. } \\
\text { - Consider patients who meet third party coverage determinations. }\end{array}$ \\
\hline $\begin{array}{l}\text { Providing oversight } \\
\text { for implementation } \\
\text { program }\end{array}$ & $\begin{array}{l}\text { The } \\
\text { multidisciplinary } \\
\text { team }\end{array}$ & $\begin{array}{l}\text { - Success of this program is the responsibility of a multidisciplinary team } \\
\text { - Pharmacists are leaders in this discipline and they play many key roles } \\
\text { - Other team members include physicians, geneticists, laboratory personnel, } \\
\text { informaticians, IT experts, nurses, patient advocates, institutional leadership, etc. }\end{array}$ \\
\hline
\end{tabular}

CPIC: Clinical Pharmacogenetics Implementation Consortium; FDA: United States Food and Drug Administration; DPWG: Dutch Pharmacogenetics Working Group; PGx: pharmacogenetics; CYP: cytochrome P450; CAP/CLIA: College of American Pathologists/ Clinical Laboratory Improvement Amendments

classified as informative, actionable, and testing recommended or required. In February 2020, the FDA published a table for pharmacogenomic associations which further delineates the type of pharmacogenetic association as it relates to efficacy, adverse events, and pharmacokinetic associations ${ }^{[10]}$. There are some overlap between CPIC guidelines and the FDA's table for pharmacogenomic associations; however several differences have been noted as well. To date, there are 41 medications that have both CPIC guidelines and FDA pharmacogenetic information. Of these 41 medications, the FDA drug-labels recommend pharmacogenetic testing for four (i.e., oxcarbazepine, azathioprine, mercaptopurine, and thioguanine), and requires testing for four more medications (i.e., abacavir, carbamazepine, ivacaftor, and rasburicase). Specifically, abacavir, carbamazepine, and mercaptopurine are supported by randomized controlled trials that documented improved clinical outcomes and cost effectiveness of pharmacogenetic testing before the initiation of these medications ${ }^{[11-13]}$. The FDA has also updated some drug labels with black box warnings for their pharmacogenetic associations such as clopidogrel, codeine, rasburicase, and pegloticase (https:// www.fda.gov/drugs/science-and-research-drugs/table-pharmacogenomic-biomarkers-drug-labeling) [Table 2].

\section{The genes}

Pharmacogenetic testing is the process of interrogating the implicated genes and variants to determine one's genotype and corresponding clinical phenotypes. The selection of the right gene(s) to test depends on the medication(s) of interest. The two dozen CPIC guidelines published to date cover 19 genes. Most early adopters of pharmacogenetics initiate their programs with the genes that encode the cytochrome P450 (CYP) 
Table 2. Drug-Gene pairs with strong FDA recommendations and the commercial laboratories that offer CLIA-certified clinical pharmacogenetic tests in the U.S.

\begin{tabular}{|c|c|c|c|}
\hline Drug & Gene(s) & US FDA PGx designation & Commercial labs that offer testing \\
\hline Clopidogrel & CYP2C19 & Boxed warning & $\begin{array}{l}\text { Fulgent Genetics; Sema4; Admera Health; RPRD } \\
\text { Diagnostics, LLC; OneOme LLC; Sanford Medical Genetics } \\
\text { Laboratory Sanford Imagenetics; Color; Pathway Genomics }\end{array}$ \\
\hline Codeine & CYP2D6 & Boxed warning & \multirow{2}{*}{$\begin{array}{l}\text { Fulgent Genetics; Sema4; OneOme LLC; Admera Health; } \\
\text { RPRD Diagnostics LLC; Sanford Medical Genetics } \\
\text { Laboratory Sanford Imagenetics; Color; Pathway Genomics }\end{array}$} \\
\hline Tramadol & CYP2D6 & Boxed warning & \\
\hline Pegloticase & G6PD & Boxed warning/testing required* & \multirow{2}{*}{$\begin{array}{l}\text { EGL Genetic Diagnostics Eurofins Clinical Diagnostics; } \\
\text { Invitae; Fulgent Genetics; Baby Genes by ArcherDX Clinical } \\
\text { Services; Sema4; RPRD Diagnostics, LLC }\end{array}$} \\
\hline Rasburicase & G6PD & Boxed warning/testing required* & \\
\hline Carbamazepine & $H L A-B^{\star} 1502$ & Boxed warning/testing required* & \multirow{3}{*}{$\begin{array}{l}\text { OneOme, LLC; Admera Health; RPRD Diagnostics, LLC; } \\
\text { Pathway Genomics }\end{array}$} \\
\hline Oxcarbazepine & $H L A-B \star 1502$ & Testing required ${ }^{\star}$ & \\
\hline Abacavir & $H L A-B^{\star} 5701$ & Boxed warning/testing required ${ }^{\star}$ & \\
\hline Azathioprine & TPMT/NUDT15 & Testing recommended & \multirow{3}{*}{$\begin{array}{l}\text { RPRD Diagnostics, LLC; Admera Health; OneOme, LLC; } \\
\text { Color }\end{array}$} \\
\hline Mercaptopurine & TPMT/NUDT15 & Testing Recommended & \\
\hline Thioguanine & TPMT/NUDT15 & Testing recommended & \\
\hline
\end{tabular}

Testing required means patients should be screened prior to initiation of therapy; Testing required* means genetically at-risk populations based on ancestries should tested prior to initiation of therapy; Testing recommended means patients who experience adverse drug reactions should be tested; "not a comprehensive list. All commercial labs listed offers CLIA-certified, state-licensed, saliva and peripheral (whole) blood specimen options, U.S.-based, clinical tests. Only laboratories indexed on Genetic Testing Registry (https://www.ncbi.nlm. nih.gov/gtr/) were considered for this table; CLIA - Clinical Laboratory Improvement Amendments

enzymes responsible for the majority of drug metabolism - namely $C Y P_{2} C 9, C_{1} P_{2} C_{1}$, and $C Y P_{2} D 6$. These three enzymes collectively metabolize about $40 \%$ of all medications ${ }^{[14]}$. Ultimately, the choice of the genes to test and when to test should be based on the nature of the practice site (pre-emptive or reactive), the clinical setting (e.g., cardiology vs. pain $v$ s. psychiatry), and the target patient populations. In preemptive testing, a testing panel that covers the common "pharmacogenes" may be considered so that information resulted will be applicable to future prescribing decisions. In reactive testing, the medication prescribed will determine the gene to be tested. Moreover, when initiating a targeted, clinical specialty-focus program (e.g., cardiology pharmacogenetics clinic), a careful review should be conducted to use panels that target genes implicated in the response to cardiovascular medications, have evidence of genetic association with medication-related outcomes, and pharmacogenetic-guided therapeutic recommendation such as clopidogrel, warfarin, and simvastatin.

Once the genes are selected for testing, a thorough review should be conducted to ensure that the test will interrogate all the relevant and actionable variants based on evidence and target population ${ }^{[15]}$. For instance, if your clinic or institution serves African ancestry patients, the ${ }^{{ }^{*}}{ }_{5},{ }^{*},{ }^{*} 8$ and ${ }^{{ }^{*}} 11$ variant alleles should be included in the test for the $\mathrm{CYP}_{2} \mathrm{C}_{9}$ gene along with the common $\star_{2}$ and ${ }^{\star_{3}}$ alleles. Moreover, clinicians should note that the designation of ${ }^{*} 1 /{ }^{*} 1$ or normal metabolizer is simply the absence of the set of interrogated variant alleles in the tested patient. Clinicians should ensure that the selected tests, whether single-gene or panel-bases: (1) cover genes that are relevant for the medications in question; and (2) interrogate a list of diverse variants in those genes that will inform therapeutic decisions for the target patient population. The Association for Molecular Pathology (AMP) advises on the minimum set of variant alleles for each gene, which is an excellent guide for those charged with selecting a pharmacogenetic test. To date, the AMP has published guidance for $\mathrm{CYP}_{2} \mathrm{C}_{1} 9^{[16]}$ and $\mathrm{CYP}_{2} \mathrm{C}^{[17]}$, and this group is planning to publish similar guidance for CYP2D6 and other "pharmacogenes".

\section{The test and the laboratory}

Many commercial laboratories have added pharmacogenetics to their test offerings. Traditionally, these laboratory-developed tests have been under the supervision of the U.S. FDA; however, in August 2020, the U.S. Department of Health and Human Services issued a statement that the FDA will not require pre- 
market review of laboratory-developed tests. Instead, they can still regulate these tests through the longer and more time consuming "notice and comment rulemaking process." This is an evolving topic and more on this regulation is anticipated ${ }^{[18]}$. These tests predominantly are only required to prove analytical validity, and the genes and variants being tested are neither regulated nor standardized. This could be problematic for accurate phenotype calling necessary for selecting the appropriate therapeutic guidance ${ }^{[15]}$. Additionally, some of the commercial laboratories may test and report on genes and variants that have questionable clinical utility based on limited evidence ${ }^{[19]}$. A threshold of evidence for reporting and actionability of the returned genotypes should be pre-defined as guided by FDA, CPIC, DPWG, and PharmGKB ${ }^{[15]}$. Additional drug-gene pairs on the panel with low evidence not meeting the threshold set by the implementation team should not be implemented. Rather, such information should be stored for future use when strength of evidence rises to actionability thresholds such as update of FDA drug labeling to include testing required, testing recommended, alternate medication or dosing changes, and/or CPIC Level A evidence designation with or without a published guideline. Implementation team members should pay careful attention to pharmacogenetics literature to track and react in a timely manner to new and updated information.

Notably, the CYP2D6 is an example of how the variability and lack of standardization across commercial labs could be demonstrated. CYP2D6 is a highly polymorphic gene with complexity demonstrated in copy number variation, gene deletion, hybrid alleles, with varying allele frequency for the variation across various ancestral populations. Variability in the method of CYP2D6 genotyping can inaccurately report the CYP2D6 phenotype if the genetic variation that is predominantly frequent in the health system is not captured, leading to erroneous prescribing actions. Several papers have instructed on the selection of genes and minimum number of variant alleles necessary for capturing an accurate phenotype ${ }^{[15,20]}$. When implementing $C_{2} P_{2}$ 6, implementers should confirm from laboratory officials, in-house or commercial, whether: (1) the test interrogates CYP2D6 alleles prevalent in the relevant patient populations; and (2) the test can detect with accuracy when haplotypes are duplicated, and if so, which haplotype/(s) is/are duplicated Lastly, implementing clinicians should be completely aware of how the laboratory translates genotypes into phenotypes. Ideally, both the genotypes of assayed variants and phenotypes should be provided to the clinician for verification and clinical interpretation.

Pharmacogenetic testing can be offered through a commercial or in-house laboratories provided they are accredited or certified by the College of American Pathologists (CAP) and the Clinical Laboratory Improvement Amendments (CLIA) ${ }^{[21]}$. Furthermore, most commercial pharmacogenetic tests are usually accompanied with a personalized report that details the variants tested for each gene, genotypes, phenotypes, and before 2019, the implicated medications along with genotype-guided therapeutic recommendations. In April 2019, the FDA issued a warning letter to Inova for marketing their pharmacogenetic tests with claims that it can predict patients' response to medications ${ }^{[22]}$. Since then, many commercial laboratories report results without a reference to the implicated medications or pharmacogenetic-guided therapeutic recommendations ${ }^{[23]}$. This development may negatively impact pharmacogenetics adoption into clinical practice and further embolden the resistance to implementation especially for clinicians who rely on the commercial laboratory reports' guidance for their therapeutic decisions. The FDA's concern that "providers may make inappropriate treatment decisions" using these reports as some laboratories report on drug-gene pairs with insufficient evidence to warrant clinical utility. However, a complete omission of genetic-guided therapeutic recommendations from these reports is not the solution. Report content should be standardized in a joint effort between relevant stakeholders such as pharmacogenetic experts (i.e., researchers and clinical implementers), laboratory officials, and authorities including the FDA. Until then, this development presents a unique opportunity for pharmacogeneticstrained pharmacists to bridge the knowledge gap. They can help prescribers decipher the clinical implications of the genetic results as well as recommend genetic-guided therapeutic alternatives and/or dose optimizations for patients. 


\section{SELECTING THE PATIENTS TO TEST}

\section{Target patient populations}

The question of "who to test" still remains an important and often uncertain one. Some may argue that choosing patients who have experienced adverse events, failed therapy, or simply focusing on "high risk patients" may be a reasonable approach ${ }^{[24]}$. Others suggest selecting patients who have an indication for a procedure for which a prescription for pharmacogenetic medication(s) can be anticipated ${ }^{[24]}$. Elective percutaneous coronary intervention (PCI) is an example of such procedures as patients are likely to be started on antiplatelet therapy for which $\mathrm{CYP}_{2} \mathrm{C}_{19}$ genotyping is important. Current data suggest that in many instances, patients who are genotyped for a certain indication such as PCI or with initiation of new medications are often prescribed other medications for which the pharmacogenetic test may also be relevant ${ }^{[25]}$. Early implementors have focused on patients who are expected to be prescribed medication with pharmacogenetic evidence such as the RIGHT patients in the Mayo Clinic and the PREDICT population as summarized by Dunnenberger et al. ${ }^{[26]}$.

Early adopters of genomic medicine and pharmacogenetics envisioned a time where genetic information will be generated on every patient, to be used along other data to individualize therapy. While this will be ideal to realize the widespread promise of precision medicine, barriers such as lack of institutional resources, cost of testing, lack of insurance coverage, and lack of infrastructure to support the use of genomic information pose a challenge to the "Genotype Everyone" approach ${ }^{[27]}$. Thus, selecting a candidate patient population to either justify payer's coverage of a pharmacogenetic test or build evidence for the utility of the data is still an approach most commonly taken by many institutions. Research investigators and implementers continue to collect data on patient outcomes, cost effectiveness, and the clinical utility of pharmacogenetic testing.

Some institutions initiated with a single drug-gene pair indicated by a clinical procedure or indication before expanding into other drug-gene pairs in the ambulatory setting ${ }^{[28,29]}$. Others start with medications with robust evidence for serious adverse events (such as thiopurine methyltransferase and thiopurines) before evolving into a preemptive testing approach ${ }^{[30]}$. Some institutions have also utilized algorithms to predict and prioritize those who would benefit the most from testing. For example, enterprise-level pharmacogenetic testing was performed by the Mayo Clinic and Vanderbilt University, both of which use a preemptive testing approach ${ }^{[31,32]}$. Through the RIGHT protocol at Mayo Clinic, a multivariate prediction algorithm identifies patients with high cardiovascular risk profile who are likely to be prescribed multiple medications with pharmacogenetic evidence, and therefore benefit from preemptive testing, was used to recruit 1000 patients ${ }^{[31,33]}$. INGENIOUS Trial at Indiana University uses a multi-gene panel to test patients who receive a first prescription for at least one of 27 medications with pharmacogenetic guidelines ${ }^{[34]}$.

Where resources are limited, clinicians may adopt the model that prioritizes patients likely to be prescribed multiple pharmacogenetic medications (e.g., mediations with CPIC guidance) as their initial roll out. A recent study investigating the utilization of pharmacogenetic medications using commercial claims of more than 100,000 patients with PCI, documented that $50 \%$ and $68 \%$ of patients were prescribed one or more drugs with pharmacogenetic evidence (CPIC level 1A or 1B) in addition to the antiplatelet therapy, over one and five years, respectively. This data suggest that a multi-gene panel testing may have a high utility in patients with PCI as these patients are frequently prescribed multiple medications with high pharmacogenetic evidence ${ }^{[25]}$. And therefore, PCI patients who are genotyped for $C_{2} P_{2} C_{1} 9$ for antiplatelet selection may be good targets for additional gene testing to guide other commonly prescribed medications in this high-risk cardiac patient population. Furthermore, a United States Department of Veterans Affairs (VA) study recently demonstrated that among over seven million veterans, 55\% were prescribed one or more CPIC level medications, with $15 \%$ and $11 \%$ being prescribed two and three of such medications, respectively ${ }^{[35]}$. The study projected that $92 \%, 26 \%$, and $4 \%$ of veterans could have been prescribed warfarin, 
simvastatin, and tramadol, respectively; while carrying actionable variants in $C_{Y} \mathrm{P}_{2} \mathrm{C} 9$ or $V K O R C 1$, $S L C O 1 B 1, C Y P 2 D 6$. These data collectively suggest that new practice sites should investigate the utilization and prevalence of medications with pharmacogenetic implications and prioritize testing patients who are commonly prescribed such medications.

Investigator-initiated studies and NIH funded pragmatic clinical trials are underway to prospectively assess clinical utility and cost effectiveness of pharmacogenetic implementation, and potentially will shed light on strategies for selecting patients. For example, the National Human Genome Research Institute (NHGRI) has recently funded two pragmatic trials named "ADOPT PGx" to investigate genotype-guided approaches for prescribing opioids and antidepressants. In addition, the ongoing Ubiquitous Pharmacogenomics (U-PGx) study in Europe plans to investigate the outcomes of preemptive pharmacogenetic testing in seven European countries ${ }^{[36]}$. Until these prospective data become available, it seems reasonable to focus implementation efforts on patients who are likely to be prescribed multiple medications with pharmacogenetic evidence, particularly those who anticipate a clinical procedure or initiation of a pharmacogenetic medication. Also, patients who have access to and can afford the test or have insurance coverage can be targeted to generate initial data to support widespread implementation. It should be noted that some commercial laboratories offer income-based payment assistance programs which provides about $50 \%-95 \%$ discount to qualifying patients in an effort to address disparities ${ }^{[37]}$. In the absence of such assistance, efforts should be made to encourage institutional pilot funding for patients who are unable to afford the test. Perhaps, there should also be federal grant funding allocated specifically for pharmacogenetic implementations in low-income populations. Moreover, it is logical that a multi-gene panel that test for genes and variants relevant for the medications commonly prescribed in this patient population, may potentially translate into a high economic value investment for the institution and third party-payers. Van Driest et al. ${ }^{[32]}$ observed that $91 \%$ of genotyped patients and $96 \%$ of African Americans carry at least one actionable variant when genotyped for five genes namely $C_{Y} P_{2} C_{19}, C Y P_{2} C 9, V K O R C 1$, thiopurine methyltransferase (TPMT), and CYP3A5. It is important to estimate the prevalence of actionable genotype variants in the health system, which largely depends on the ancestral distribution of the patients. Implementers have either used genotype data generated for their population ${ }^{[32,38]}$ or projected the rate of actionable genotypes using published population frequency from CPIC and 1000 Genomes, among others $^{[35]}$. Of note, the frequency of actionable phenotypes may be higher if concomitant administration of enzyme (i.e., CYP2D6, CYP2C19, etc.) inhibitors is considered. Mostafa et al. ${ }^{[39]}$ recently documented that the prevalence $C Y P_{2} D 6$ and $C Y P_{2} C_{1} 9$ poor metabolizer phenotypes were increased by fivefold after considering co-medication with substrates that can inhibit these enzymes among 5,408 Australians. More on enzyme inhibitors is discussed below in the phenoconversion section.

Surveys are valuable to understanding implementation challenges and work out solutions to overcome encountered barriers. A recent paper that surveyed the physicians within a pharmacogenetic implementation program reported that having genotype data in the EHR before a patient-physician encounter is critical for effective implementation ${ }^{[28]}$. This suggests focusing on patients who are anticipated to have pre-appointment visits where patients can be genotyped, and information is returned to EHR before a physician encounter. In addition, an approach that targets patients scheduled for elective arthroplasty for CYP2D6-guided opioid prescribing may prove to be successful. Patients in this setting can be genotyped at a pre-operative visit with the results returned in the EHR for real-time use and effective genotypeguided prescribing ${ }^{[40]}$. Within the primary care setting, patients who are predicted to start a medication with pharmacogenetic recommendations may also be targeted for pharmacogenetic testing. If the genotype cannot be made available before the patient-physician encounter, discussions should be made around the willingness of physicians and patients to wait for the genotyping results before prescribing the medication. This was the case in a recent pragmatic clinical trial of $\mathrm{CYP}_{2} \mathrm{C}_{1} 9$ implementation for proton pump inhibitor use in pediatrics, where patients and physicians were willing to wait on the genotyping results for genotype- 
guided proton pump inhibitor prescribing ${ }^{[41]}$. Although patients can be started on a medication before the return of genotyping results, this is not an ideal model, and may result in patients being lost to follow up with increases in economic burden for patients and healthcare system due to the costs of treatment for adverse drug reactions, treatment failures and even the cost of the wasted initially filled medication.

\section{PROVIDING OVERSIGHT FOR IMPLEMENTATION PROGRAM}

\section{The multidisciplinary implementation team}

Several academic and non-academic institutions have spearheaded pharmacogenetic implementation efforts, documenting site-specific implementation experiences and lessons learned. These implementation efforts were summarized in other review papers ${ }^{[26,42]}$. Many of the implementation services were pharmacist-led ${ }^{[43-45]}$. Pharmacists play many key roles in the implementation of pharmacogenetics such as reviewing medication use and safety policies, developing educational strategies for other providers and patients, reviewing and analyzing evidence, managing the pharmacogenetic database, and implementing overall leadership ${ }^{[43]}$. Physicians are also critical to the development and success of the implementation. Understanding their interest within a practice site and how they perceive pharmacogenetics in their clinical workflow is important for successful integration. Structured or semi-structured surveys administered at the pre-implementation stage may help implementing clinicians glean understanding of the institutional landscape, stakeholders' goals, and clinicians' interest and their perspectives about the use of pharmacogenetics ${ }^{[46]}$. Physician-champions should be identified and in the planning process for the targeted clinical group and/or specialty. Their clinical expertise is vital especially when making protocol decisions on pharmacogenetic-guided therapeutic recommendations and their associated alternative therapies or dosing. Like pharmacists, physicians should be included in the oversight committee that is established early on to oversee the activities from the planning phase through implementation of clinical pharmacogenetics and post-implementation evaluation. Already established Pharmacy and Therapeutics (P\&T) Committees can serve this purpose as well. Additionally, experts from genetics, clinical laboratory, informatics and information technology, administration, institutional leadership and patient advocacy should be included in this committee.

\section{RELEVANT RESOURCES FOR PHARMACOGENETIC IMPLEMENTATION}

\section{Clinical Pharmacogenetics Implementation Consortium}

The CPIC is an international consortium of volunteers and dedicated members who are interested in facilitating the use of pharmacogenetic information for patient care. It was created in 2009 as a shared project between the international pharmacogenetics database and website, PharmGKB (www.pharmgkb. org) and the NIH. The purpose of CPIC is to provide freely available, peer-reviewed, evidence-based clinical pharmacogenetic practice guidelines. As of 2019, CPIC has a growing membership of 350 members from 245 institutions in 33 countries with observers from the U.S. FDA and NIH. Members meet monthly on general membership calls and through the informatics, dissemination, and other workgroup meetings. CPIC guidelines are indexed in PubMed and published on the CPIC website (https://cpicpgx.org/ guidelines/) as well as annotated on the PharmGKB website (www.pharmgkb.org). As of August 2020, there are 24 published guidelines with 2 more in progress. Their goal is to update the guidelines on a biennial schedule. So far, they cover 19 genes and 46 medications across several therapeutic areas including infectious diseases, cardiology, psychiatry, neurology, and oncology. CPIC also compiles a list of contacts for PGx implementers across the U.S. (https://cpicpgx.org/implementation/) and other countries, which is a great resource of contacts for experts and clinicians in the field.

\section{United States Food and Drug Administration}

Through their oversight of drug product labeling, the FDA also maintains a table of pharmacogenomic biomarkers (https://www.fda.gov/drugs/science-and-research-drugs/table-pharmacogenomic-biomarkers- 
drug-labeling). In February 2020, they published a table of pharmacogenetic associations (https://www. fda.gov/medical-devices/precision-medicine/table-pharmacogenetic-associations) to highlight druggene associations that are likely to have altered metabolism and, in certain cases, differential therapeutic effects such as risks for adverse events and therapeutic failures. This is a living document and the FDA has an open docket for stakeholders to offer specific comments about inclusion of other pharmacogenetic associations along with the rationale and supportive evidence. It is unclear at this point how often this table will be updated. While CPIC has published their process for robust and systematic evidence review to develop their peer-reviewed guidelines ${ }^{[47]}$; the FDA does not explicitly discuss the evidence base underlying the recommendations presented in their table of pharmacogenetic associations.

\section{Dutch Pharmacogenetics Working Group}

In 2005, the Royal Dutch Pharmacists Association established the DPWG with the objective of developing pharmacogenomic-based therapeutic and dose recommendations. Thus far, recommendations have been developed for over 80 drugs covering various therapeutic classes covered in both the CPIC and FDA databases. These guidelines are updated every 3 months and are available to all physicians and pharmacists in the Netherlands. They have also been integrated into the clinical decision support systems in the Netherlands and serve as the main source of pharmacogenetic-guided recommendations used in U-PGx in Europe $e^{[6,36]}$.

Of note, the FDA, CPIC, and DPWG guidelines offer similar recommendations for most drug-gene pairs; however, differences in the guidelines' development processes and/or allele and phenotype classifications have resulted in discordance in some recommendations ${ }^{[48]}$. For instance, pharmacogeneticguided recommendations for aripiprazole, clozapine, and gefitinib, among others, vary across all three platforms. CPIC and DPWG are currently working on standardization of alleles and phenotypes ${ }^{[49]}$. The recent $C Y P_{2} D 6$ genotype-phenotype term standardization project is one of the most important efforts in this area ${ }^{[50]}$. Using standardized terms and harmonizing the guidelines are likely to facilitate clinical implementation efforts and data sharing across health systems around the world.

\section{The Canadian Pharmacogenomics Network for Drug Safety}

The Canadian Pharmacogenomics Network for Drug Safety (CPNDS) is an innovative, national program that aims to reduce serious adverse drug reactions in children and adults ${ }^{[51]}$. They have developed their own specific "drug biotransformation" testing panel which contains variants of genes that are known to influence the way a patient responds to a given medication or may predispose a patient to an adverse drug reaction. Having a national health care system in Canada with an active surveillance network facilitates the integration and dissemination of CPNDS guidelines to accelerate adoption of pharmacogenetics.

\section{OTHER PERTINENT CLINICAL CONSIDERATIONS}

Assessment of pharmacogenetic results for clinical applications should also account for non-genetic clinical and environmental factors to evade incomplete conclusions and misguided recommendations. Drugdrug interactions, disease states, organ function, hormonal status, and considerations of additional patient specific factors are regularly taken into consideration for medication and dose selection. Drug-druggene interactions should be duly considered as the efficacy and incidence of adverse events are chiefly the result of parent compound and metabolite concentrations. In this section, we will review the concept of phenoconversion, considerations for switching medications, and guidance for special populations including transplant and pediatric patients as well as pregnant women. Other disease states have also been found to modulate the activity of metabolizing enzymes, though with less clarity for clinical actionability and therefore will not be covered here ${ }^{[52]}$. 


\section{Selecting an alternative therapy based on pharmacogenetic results}

As with any therapy selection, prior patient experience with the medication, adherence, and cost should be taken into consideration. Apart from the usual clinical considerations for prescribing such as age, organ function (i.e., kidney, liver, etc.), co-medications, and others, close attention should be paid to these three principles when selecting an alternative therapy based on pharmacogenetic results - (1) the indication for the medication; (2) pharmacogenetic implications for the alternative therapy; and (3) loading dose requirements. Some pharmacogenetic recommendations are indication specific because the evidence for clinical actionability has been consistently replicated in select patient populations. This is the case with clopidogrel and $\mathrm{CYP}_{2} \mathrm{C}_{1} 9$ intermediate and poor metabolizers who have acute coronary syndrome and have had a PCI. Although clopidogrel has other indications, only alternative therapies that produce antiplatelet effects for post-PCI patients should be considered in this population (i.e., prasugrel or ticagrelor). Secondly, clinicians must also consider the pharmacogenetic influences with the alternative medication and ensure that patients do not carry high risk variants for the alternative agents. For example, palonosetron would not be an ideal alternative for $C_{Y} P_{2} D 6$ ultrarapid metabolizers who are not responsive to ondansetron since it is also a substrate of the CYP2D6 metabolic pathway. Of note, the CPIC guideline incorporates this consideration into their therapeutic recommendations. Lastly, clinicians must confirm whether loading doses should be administered when making the switch to an alternative medication. For instance, when switching from clopidogrel to prasugrel or ticagrelor within the first 30 days postPCI, the use of $60-\mathrm{mg}$ or $180-\mathrm{mg}$ loading dose, respectively, has been shown to be beneficial. However, it is reasonable to omit the loading dose if switching beyond 30 days from $\mathrm{PCI}^{[53]}$. Many of institutions who implemented pharmacogenetics rely on trained pharmacists to evaluate the pharmacogenetic results and recommend changes to the prescribing clinician. These recommendations are better implemented if they are communicated through consult notes with specific recommendations in concise format. Interventions are likely to happen if clinicians have a chance for a patient encounter after the pharmacogenetic results are reported in EHR.

\section{Phenoconversion}

Pharmacogenetic results can be applicable throughout a patient's lifetime depending on the variants interrogated and the genotype result. However, extrinsic factors such as concomitant medications can lead to a transient discordance between the genotype and its corresponding phenotype - this concept is termed as "phenoconversion" or "drug-drug-gene interaction" ${ }^{[54]}$. Currently, evidence shows CYP2D6, CYP2C19, CYP2C9, and TPMT are enzymes susceptible to phenoconversion ${ }^{[5]}$. These enzymes are subject to inhibition and induction (except for $C Y P_{2} D 6$ which is not inducible) by medications and thus concomitant medications administered for patients must be interrogated for phenoconversion. Phenoconversion is most commonly seen with patients carrying alleles encoding for functional enzymes. For example, genetic results interpreted as a $\mathrm{CYP}_{2} \mathrm{D}_{6}$ normal metabolizer can phenotypically present as a $C Y P_{2} D 6$ poor metabolizer due to the presence of strong $C Y P_{2} D 6$ inhibitor (e.g., paroxetine or fluoxetine ${ }^{[56]}$. In general, poor and ultrarapid metabolizers (of $C Y P_{2} C_{19}, C Y P_{2} C$ 9, and $C Y P_{2} D 6$ ) are less likely to be affected by this phenomenon, but the clinical impact will depend on the co-administered drugs ${ }^{[57]}$. Poor metabolizers carry two alleles that produce decreased or nonfunctional enzymes, thus strong inhibition and induction may not affect the functional activity of the enzyme. For ultrarapid metabolizers, or carriers of increased transcription alleles or gene duplication, the risk of phenoconversion is less likely but still possible with strong inhibitors and substrate medications highly dependent on the enzyme ${ }^{[57,58]}$. Lastly, considerations of how dependent the substrate medication is on the enzyme pathway (e.g., major or minor pathway) will determine the relevance of the gene and therefore the likelihood of phenoconversion being clinically relevant. Alternative metabolic pathways, for patient encoding functional enzymes, will decrease the clinical impact of an inhibitor or inducer for patients. Clinically relevant strong inhibitors should be monitored when being prescribed with other substrates of the same enzyme pathway. In this section we will discuss common clinically actionable drug-drug-gene interactions examples with $C Y P_{2} D 6, C Y P_{2} C_{19}$, CYP2C9, and TPMT. 
CYP2D6

For the highly polymorphic $C Y P_{2} D 6$ gene, calculation of an activity score is used to translate genetic variants to phenotype (e.g., metabolizer status) ${ }^{[59]}$. The CPIC guidelines have addressed incorporation of an inhibitor drug when calculating activity scores based on a system used for tamoxifen in breast cancer patient study ${ }^{[60,61]}$. Substrates of CYP2D6 include propafenone, paroxetine, risperidone, codeine, tramadol, and atomoxetine ${ }^{[62]}$. When concomitant strong $C Y_{2}{ }_{2} 6$ inhibitors are present, the activity score is multiplied by zero and the resultant phenotype is a poor metabolizer while for moderate to weak inhibitors the activity score is halved, and so the implicated phenotype will vary. For example, paroxetine significantly inhibits flecainide, desipramine, aripiprazole, and R-methadone metabolism in individuals carrying two functional alleles than those with decreased function alleles ${ }^{[55]}$. In addition, since herbal supplements are also metabolized via the CYP450 enzyme system and can cause phenoconversion, patients should be cautioned to report all active herbal supplement usage. Supplements such as bush mint, bush tea, or pignut should be monitored for their inhibitory effects on $C Y P_{1} A_{2}, C Y P_{3} A 4$, and especially $C Y P_{2} D_{6}{ }^{[52]}$.

\section{$\mathrm{CYP}_{2} \mathrm{C} 19$}

The interaction between proton pump inhibitors and clopidogrel is attributed to inhibition of $\mathrm{CYP}_{2} \mathrm{C}_{1}$, however this has not been found to be a class effect by all proton pump inhibitors. Their influence on clopidogrel bioactivation differs by the agent and by the CYP $2 C_{1} 9$ metabolizer status of the patient. Omeprazole, esomeprazole, and rabeprazole demonstrate stronger competitive $\mathrm{CYP}_{2} \mathrm{C}_{19} 9$ inhibition than lansoprazole and have significant impact in patients who are carriers of functional or increased function alleles (i.e., $\mathrm{CYP}_{2} \mathrm{C}_{1} 9$ normal, rapid and ultrarapid metabolizers) compared to carriers of loss of function alleles (i.e., $C Y P_{2} C_{1} 9$ intermediate and poor metabolizers) ${ }^{[55,63]}$. A prospective double-blind, crossover, randomized study, found that the use of esomeprazole significantly reduced platelet inhibition in clopidogrel-treated acute coronary syndrome patients who were carriers of ${ }^{*} 17$ or ${ }^{*} 1{ }^{*} 1$ compared with those treated with clopidogrel and placebo. This interaction was not observed among ${ }^{{ }^{*}}$ carriers $^{[64]}$.

\section{$\mathrm{CYP}_{2} \mathrm{C} 9$}

Clinically relevant inhibitors of $\mathrm{CYP}_{2} \mathrm{C} 9$ include amiodarone, fluconazole, and miconazole. Coadministration of warfarin ( $\mathrm{CYP}_{2} \mathrm{C} 9$ substrate) and amiodarone ( $\mathrm{CYP}_{2} \mathrm{C} 9$ inhibitor) require adjustment of dose, which is accounted for in warfarin pharmacogenetic dosing algorithms ${ }^{[65,66]}$. Likewise, fluconazole, an inhibitor of $\mathrm{CYP}_{2} \mathrm{C} 9$ among other enzymes, produced a 2 -fold increase in siponimod plasma concentrations among $\mathrm{CY}_{2} \mathrm{C} 9$ normal metabolizers. Notably, normal metabolizers of $\mathrm{CYP}_{2} \mathrm{C} 9$ can be phenoconverted to intermediate or poor metabolizers ${ }^{[67,68]}$. Commonly prescribed CYP2C9 substrates like warfarin, phenytoin, proton pump inhibitors, and non-steroidal ant-inflammatory drugs should be monitored for phenoconversion ${ }^{[62]}$.

\section{Thiopurine Methyltransferase}

TPMT activity is important for substrates including mercaptopurine, azathioprine, thioguanine, and cisplatin ${ }^{[69,70]}$. This enzyme's activity is affected by recent blood transfusions for which enzyme activity testing alone may result in inaccurate results unlike pharmacogenetic testing, which remains stable despite transfusion ${ }^{[71]}$. However, co-administration with additional bone marrow suppressing drugs such as sulfasalazine, mesalamine, and olsalazine should be carefully monitored and incorporated into the interpretation of pharmacogenetic results ${ }^{[54]}$.

Moreover, social habits should also be taken into consideration. For instance, smoking induces CYP1A2 which may be relevant for drugs affected by $C Y P_{1} A 2$ such as caffeine, clozapine, fluvoxamine, olanzapine, and theophylline ${ }^{[72]}$. Phenoconversion is not permanent so once the interacting medication or habit is discontinued, the pharmacogenetic-guided therapeutic recommendation should be re-examined and corrected as necessary. 


\section{Organ transplant patients}

In cases of stem cell, liver, and bowel transplant, special consideration should be taken when collecting and interpreting pharmacogenetic results. Clinicians must ensure that samples from stem cell transplant patients are collected prior to the transplant for DNA analysis. This guarantees that the transplant recipient's DNA is analyzed and not that of the transplant donor. Moreover, the liver and intestines possess CYP3A enzymes important for metabolism of many medications including tacrolimus. In addition, preliminary studies have found that formulation and route of tacrolimus influences the significance of CYP $3 A 5$ pharmacogenetic results ${ }^{[73,74]}$. When evaluating pharmacogenetic results for liver and bowel transplant recipients; clinicians should be aware that the genetic makeup of the new graft (i.e., donor's genotype) may be different from that of the transplant recipient. Hence, the use of only the recipient's genetic information may be misleading. Studies are underway to help unravel the pharmacogenetic complexities related to the type of transplant and the influence of the recipient versus the donor's genotypes as it relates to the timing of the transplant surgery and beyond.

\section{Ontogeny}

Neonatal and pediatric patients undergo age related development of metabolizing enzymes. For this reason, a general knowledge of the enzymes' maturation process is discussed here. A simplified categorization of the process of maturation can be grouped into three categories ${ }^{[75]}$. The first are enzymes that are expressed at high levels during gestation and within 1-2 years after birth with CYP3A7 being an example of this category. The second category comprises $C_{3} P_{3} A_{5}$ and $C_{1} P_{2} C_{1} 9$ which are expressed at constant levels during gestation. Unlike $C Y P_{3} A 5, C Y P_{2} C_{19}$ 's expression increases throughout the first year of life. The third category are those enzymes that are not expressed or are at low levels during gestation and increase substantially during the first to second years of birth. Examples are CYP3A4, CYP2C9, CYP2D6, and

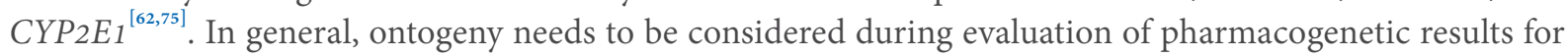
neonates, infants, and younger pediatric patients.

\section{Pregnancy}

Pregnancy results in a multitude of endocrine as well as metabolic changes that affect interpretation of pharmacogenetic results. Most CYP450 enzymes are induced during pregnancy namely $C Y P 2 A 6$, $C Y P_{2} B 6, C Y P_{2} C 8, C Y P_{2} C 9, C Y P_{2} E_{1}, C Y P_{3} A 4$, and $C Y P_{3} A 5^{[76]}$. CYP2D6 enzyme levels are induced to higher levels in the second through third trimester for ultrarapid and normal metabolizers compared to first trimester ${ }^{[77,78]}$. Clinically, this indicates that normal metabolizers of these enzymes would need monitoring for the duration of the pregnancy to ensure efficacy with active drugs such as antidepressants and antiemetics, and safety with prodrugs such as codeine, since pregnancy may induce CYP2D6, leading to a potential change in metabolizer status, affecting medications that are metabolized through $C Y P_{2} D 6^{[57,79,60]}$. However, for pregnant women who are ultrarapid metabolizers, no further adjustments to the actionable pharmacogenetic recommendations would be anticipated. For instance, ondansetron would not be recommended for any $C Y P_{2} D 6$ ultra-rapid metabolizer regardless of pregnancy status ${ }^{[77-79]}$. $C Y P_{2} D 6$ intermediate and poor metabolizers need no additional adjustments from guideline recommendations during pregnancy ${ }^{[77,78]}$. Alternatively, inhibitory effects on $C Y P_{1} A_{2}$ and $C Y P_{2} C_{19}$ enzyme levels have also been reported during pregnancy ${ }^{[77,80]}$. Use of caffeine (a substrate of CYP1A2) in the third trimester results in a $65 \%$ decreased metabolism as compared to non-pregnant women ${ }^{[77]}$. The current limited evidence in pregnant women suggest that it is advisable to monitor normal metabolizers in addition to other actionable phenotypes to ensure that all clinically-relevant factors are carefully considered when utilizing pharmacogenetic results to make therapeutic decisions in pregnancy.

\section{CONCLUSION}

In summary, pharmacogenetic testing is a valuable tool that should be adopted into routine clinical practice. We provide a review of the preparatory steps necessary to implement a clinical pharmacogenetic 
program and other pertinent clinical considerations to maximize the impact of pharmacogenetic testing in clinical practice [Table 1]. We comment on choosing the gene(s) and variants to test, the patient population, available resources, and the impact of phenoconversion, organ transplant, and pregnancy on pharmacogenetic applications. At its core, it is about the individual and how best we can optimize therapeutic recommendations for each patient. Yet, the achievement of this goal is largely driven by a team effort in which pharmacists play vital roles. Pharmacogenetics is not the ultimate decision maker when it comes to making prescribing decisions. Other intrinsic and extrinsic factors should also be considered along with the pharmacogenetic information to make an informed decision on the optimal therapeutic regimen for each patient.

\section{DECLARATIONS}

\section{Authors' contributions}

Made substantial contributions to conception and design of the paper: Owusu Obeng A, El Rouby N, Liu M, and Wallsten $\mathrm{R}$

Provided administrative support: Owusu Obeng A, El Rouby N

Provided technical support: Owusu Obeng A, El Rouby N, Liu M, Wallsten R

Contributed to the writing, review and final approval of the manuscript: Owusu Obeng A, El Rouby N, Liu $\mathrm{M}$, Wallsten $\mathrm{R}$

\section{Availability of data and materials}

Not applicable.

\section{Financial support and sponsorship}

Owusu Obeng A was partially funded under NHGRI (3U01HG008701) during the development of this manuscript. Liu M was partially funded under NHGRI (U01HG010232) during the development of this manuscript. Wallsten $\mathrm{R}$ is an employee of Sema4.

\section{Conflicts of interest}

All authors declared that there are no conflicts of interest.

\section{Ethical approval and consent to participate}

Not applicable.

\section{Consent for publication}

Not applicable.

\section{Copyright}

(c) The Author(s) 2021.

\section{REFERENCES}

1. Smith DM, Weitzel KW, Elsey AR, et al. CYP2D6-guided opioid therapy improves pain control in CYP2D6 intermediate and poor metabolizers: a pragmatic clinical trial. Genet Med 2019;21:1842-50.

2. Coenen MJ, de Jong DJ, van Marrewijk CJ, et al; TOPIC Recruitment Team. Identification of Patients With Variants in TPMT and Dose Reduction Reduces Hematologic Events During Thiopurine Treatment of Inflammatory Bowel Disease. Gastroenterology 2015;149:90717.e7.

3. Pirmohamed M. Personalized pharmacogenomics: predicting efficacy and adverse drug reactions. Annu Rev Genomics Hum Genet 2014;15:349-70.

4. Caudle KE, Klein TE, Hoffman JM, et al. Incorporation of pharmacogenomics into routine clinical practice: the Clinical Pharmacogenetics Implementation Consortium (CPIC) guideline development process. Curr Drug Metab 2014;15:209-17.

5. Hoffman JM, Dunnenberger HM, Kevin Hicks J, et al. Developing knowledge resources to support precision medicine: principles from 
the Clinical Pharmacogenetics Implementation Consortium (CPIC). J Am Med Inform Assoc 2016;23:796-801.

6. Swen JJ, Nijenhuis M, de Boer A, et al. Pharmacogenetics: from bench to byte--an update of guidelines. Clin Pharmacol Ther 2011;89:662-73.

7. Manolio TA, Rowley R, Williams MS, et al. Opportunities, resources, and techniques for implementing genomics in clinical care. Lancet 2019;394:511-20.

8. Peterson JF, Field JR, Shi Y, et al. Attitudes of clinicians following large-scale pharmacogenomics implementation. Pharmacogenomics $J$ 2016;16:393-8.

9. Frigon MP, Blackburn MÈ, Dubois-Bouchard C, Gagnon AL, Tardif S, Tremblay K. Pharmacogenetic testing in primary care practice: opinions of physicians, pharmacists and patients. Pharmacogenomics 2019;20:589-98.

10. FDA, U. Table of Pharmacogenetic Associations. Available from: https://www.fda.gov/medical-devices/precision-medicine/tablepharmacogenetic-associations. [Last accessed on 16 Dec 2020]

11. Schackman BR, Scott CA, Walensky RP, Losina E, Freedberg KA, Sax PE. The cost-effectiveness of HLA-B*5701 genetic screening to guide initial antiretroviral therapy for HIV. AIDS 2008;22:2025-33.

12. Plumpton CO, Pirmohamed M, Hughes DA. Cost-Effectiveness of Panel Tests for Multiple Pharmacogenes Associated With Adverse Drug Reactions: An Evaluation Framework. Clin Pharmacol Ther 2019;105:1429-38.

13. Choi H, Mohit B. Cost-effectiveness of screening for HLA-B*1502 prior to initiation of carbamazepine in epilepsy patients of Asian ancestry in the United States. Epilepsia 2019;60:1472-81.

14. Vo TT, Bell GC, Owusu Obeng A, Hicks JK, Dunnenberger HM. Pharmacogenomics Implementation: Considerations for Selecting a Reference Laboratory. Pharmacotherapy 2017;37:1014-22.

15. Bousman CA, Zierhut H, Müller DJ. Navigating the Labyrinth of Pharmacogenetic Testing: A Guide to Test Selection. Clin Pharmacol Ther 2019;106:309-12.

16. Pratt VM, Del Tredici AL, Hachad H, et al. Recommendations for Clinical CYP2C19 Genotyping Allele Selection: A Report of the Association for Molecular Pathology. J Mol Diagn 2018;20:269-76.

17. Pratt VM, Cavallari LH, Del Tredici AL, et al. Recommendations for Clinical CYP2C9 Genotyping Allele Selection: A Joint Recommendation of the Association for Molecular Pathology and College of American Pathologists. J Mol Diagn 2019;21:746-55.

18. Rescission of Guidances and Other Informal Issuances Concerning Premarket Review of Laboratory Developed Tests. Available from: https://www.hhs.gov/coronavirus/testing/recission-guidances-informal-issuances-premarket-review-lab-tests/index.html. [Last accessed on 16 Dec 2020]

19. de Leon J. Have we successfully implemented CYP2D6 genotyping in psychiatry? Expert Opin Drug Metab Toxicol 2017;13:1201-3.

20. Bousman C, Maruf AA, Müller DJ. Towards the integration of pharmacogenetics in psychiatry: a minimum, evidence-based genetic testing panel. Curr Opin Psychiatry 2019;32:7-15.

21. Arwood MJ, Chumnumwat S, Cavallari LH, Nutescu EA, Duarte JD. Implementing Pharmacogenomics at Your Institution: Establishment and Overcoming Implementation Challenges. Clin Transl Sci 2016;9:233-45.

22. Warning Letter Inova Genomics Laboratory. Available from: https://www.fda.gov/inspections-compliance-enforcement-and-criminalinvestigations/warning-letters/inova-genomics-laboratory-577422-04042019. [Last accessed on 16 Dec 2020]

23. FDA Stepping Up Actions Against PGx Testing, Forcing Some Labs to Stop Reporting Drug Information. Available from: https:// www.360dx.com/regulatory-news-fda-approvals/fda-stepping-actions-against-pgx-testing-forcing-some-labs-stop\#.X7Kz8ZNKjRY. [Last accessed on 16 Dec 2020]

24. Roden DM, Van Driest SL, Mosley JD, et al. Benefit of Preemptive Pharmacogenetic Information on Clinical Outcome. Clin Pharmacol Ther 2018;103:787-94.

25. El Rouby N, Alrwisan A, Langaee T, et al. Clinical Utility of Pharmacogene Panel-Based Testing in Patients Undergoing Percutaneous Coronary Intervention. Clin Transl Sci 2020;13:473-81.

26. Dunnenberger HM, Crews KR, Hoffman JM, et al. Preemptive clinical pharmacogenetics implementation: current programs in five US medical centers. Аппи Rev Pharmacol Toxicol 2015;55:89-106.

27. Manolio TA, Chisholm RL, Ozenberger B, et al. Implementing genomic medicine in the clinic: the future is here. Genet Med 2013;15:258-67.

28. Cicali EJ, Weitzel KW, Elsey AR, et al. Challenges and lessons learned from clinical pharmacogenetic implementation of multiple genedrug pairs across ambulatory care settings. Genet Med 2019;21:2264-74.

29. Cavallari LH, Weitzel KW, Elsey AR, et al. Institutional profile: University of Florida Health Personalized Medicine Program. Pharmacogenomics 2017;18:421-6.

30. Hoffman JM, Haidar CE, Wilkinson MR, et al. PG4KDS: a model for the clinical implementation of pre-emptive pharmacogenetics. $\mathrm{Am}$ J Med Genet C Semin Med Genet 2014;166C:45-55.

31. Bielinski SJ, Olson JE, Pathak J, et al. Preemptive genotyping for personalized medicine: design of the right drug, right dose, right timeusing genomic data to individualize treatment protocol. Mayo Clin Proc 2014;89:25-33.

32. Van Driest SL, Shi Y, Bowton EA, et al. Clinically actionable genotypes among 10,000 patients with preemptive pharmacogenomic testing. Clin Pharmacol Ther 2014;95:423-31.

33. Bielinski SJ, St Sauver JL, Olson JE, et al. Cohort Profile: The Right Drug, Right Dose, Right Time: Using Genomic Data to Individualize Treatment Protocol (RIGHT Protocol). Int J Epidemiol 2020;49:23-24k.

34. Rosenman MB, Decker B, Levy KD, Holmes AM, Pratt VM, Eadon MT. Lessons Learned When Introducing Pharmacogenomic Panel Testing into Clinical Practice. Value Health 2017;20:54-9. 
35. Chanfreau-Coffinier C, Hull LE, Lynch JA, et al. Projected Prevalence of Actionable Pharmacogenetic Variants and Level A Drugs Prescribed Among US Veterans Health Administration Pharmacy Users. JAMA Netw Open 2019;2:e195345.

36. Cecchin E, Roncato R, Guchelaar HJ, Toffoli G; Ubiquitous Pharmacogenomics Consortium. Ubiquitous Pharmacogenomics (U-PGx): The Time for Implementation is Now. An Horizon2020 Program to Drive Pharmacogenomics into Clinical Practice. Curr Pharm Biotechnol 2017;18:204-9.

37. L Rogers S, Keeling NJ, Giri J, et al. PARC report: a health-systems focus on reimbursement and patient access to pharmacogenomics testing. Pharmacogenomics 2020;21:785-96.

38. Ji Y, Skierka JM, Blommel JH, et al. Preemptive Pharmacogenomic Testing for Precision Medicine: A Comprehensive Analysis of Five Actionable Pharmacogenomic Genes Using Next-Generation DNA Sequencing and a Customized CYP2D6 Genotyping Cascade. $J$ Mol Diagn 2016;18:438-45.

39. Mostafa S, Kirkpatrick CMJ, Byron K, Sheffield L. An analysis of allele, genotype and phenotype frequencies, actionable pharmacogenomic (PGx) variants and phenoconversion in 5408 Australian patients genotyped for CYP2D6, CYP2C19, CYP2C9 and VKORC1 genes. J Neural Transm (Vienna) 2019;126:5-18.

40. Cavallari LH, Johnson JA. A case for genotype-guided pain management. Pharmacogenomics 2019;20:705-8.

41. Cicali EJ, Blake K, Gong Y, et al. Novel Implementation of Genotype-Guided Proton Pump Inhibitor Medication Therapy in Children: A Pilot, Randomized, Multisite Pragmatic Trial. Clin Transl Sci 2019;12:172-9.

42. Volpi S, Bult CJ, Chisholm RL, et al. Research Directions in the Clinical Implementation of Pharmacogenomics: An Overview of US Programs and Projects. Clin Pharmacol Ther 2018;103:778-86.

43. Owusu-Obeng A, Weitzel KW, Hatton RC, et al. Emerging roles for pharmacists in clinical implementation of pharmacogenomics. Pharmacotherapy 2014;34:1102-12.

44. Johnson JA. Pharmacists should jump onto the clinical pharmacogenetics train. Am J Health Syst Pharm 2016;73:2013-6.

45. Weitzel KW, Elsey AR, Langaee TY, et al. Clinical pharmacogenetics implementation: approaches, successes, and challenges. Am J Med Genet C Semin Med Genet 2014;166C:56-67.

46. Rafi I, Crinson I, Dawes M, Rafi D, Pirmohamed M, Walter FM. The implementation of pharmacogenomics into UK general practice: a qualitative study exploring barriers, challenges and opportunities. J Community Genet 2020;11:269-77.

47. Caudle KE, Gammal RS, Whirl-Carrillo M, Hoffman JM, Relling MV, Klein TE. Evidence and resources to implement pharmacogenetic knowledge for precision medicine. Am J Health Syst Pharm 2016;73:1977-85.

48. Bank PCD, Caudle KE, Swen JJ, et al. Comparison of the Guidelines of the Clinical Pharmacogenetics Implementation Consortium and the Dutch Pharmacogenetics Working Group. Clin Pharmacol Ther 2018;103:599-618.

49. Caudle KE, Dunnenberger HM, Freimuth RR, et al. Standardizing terms for clinical pharmacogenetic test results: consensus terms from the Clinical Pharmacogenetics Implementation Consortium (CPIC). Genet Med 2017;19:215-23.

50. Caudle KE, Sangkuh1 K, Whirl-Carrillo M, et al. Standardizing CYP2D6 Genotype to Phenotype Translation: Consensus Recommendations from the Clinical Pharmacogenetics Implementation Consortium and Dutch Pharmacogenetics Working Group. Clin Transl Sci 2020;13:116-24.

51. Ross CJ, Visscher H, Sistonen J, et al; CPNDS Consortium. The Canadian Pharmacogenomics Network for Drug Safety: a model for safety pharmacology. Thyroid 2010;20:681-7.

52. Gaedigk A, Dinh JC, Jeong H, Prasad B, Leeder JS. Ten Years' Experience with the CYP2D6 Activity Score: A Perspective on Future Investigations to Improve Clinical Predictions for Precision Therapeutics. J Pers Med 2018;8:15.

53. Angiolillo DJ, Rollini F, Storey RF, et al. International Expert Consensus on Switching Platelet $\mathrm{P} 2 \mathrm{Y}_{12}$ Receptor-Inhibiting Therapies. Circulation 2017;136:1955-75.

54. Shah RR, Smith RL. Addressing phenoconversion: the Achilles' heel of personalized medicine. Br J Clin Pharmacol 2015;79:222-40.

55. Bahar MA, Setiawan D, Hak E, Wilffert B. Pharmacogenetics of drug-drug interaction and drug-drug-gene interaction: a systematic review on CYP2C9, CYP2C19 and CYP2D6. Pharmacogenomics 2017;18:701-39.

56. Storelli F, Matthey A, Lenglet S, Thomas A, Desmeules J, Daali Y. Impact of CYP2D6 Functional Allelic Variations on Phenoconversion and Drug-Drug Interactions. Clin Pharmacol Ther 2018;104:148-57.

57. Hicks JK, Bishop JR, Sangkuhl K, et al; Clinical Pharmacogenetics Implementation Consortium. Clinical Pharmacogenetics Implementation Consortium (CPIC) Guideline for CYP2D6 and CYP2C19 Genotypes and Dosing of Selective Serotonin Reuptake Inhibitors. Clin Pharmacol Ther 2015;98:127-34.

58. Lam YW, Gaedigk A, Ereshefsky L, Alfaro CL, Simpson J. CYP2D6 inhibition by selective serotonin reuptake inhibitors: analysis of achievable steady-state plasma concentrations and the effect of ultrarapid metabolism at CYP2D6. Pharmacotherapy 2002;22:1001-6.

59. Gaedigk A, Simon SD, Pearce RE, Bradford LD, Kennedy MJ, Leeder JS. The CYP2D6 activity score: translating genotype information into a qualitative measure of phenotype. Clin Pharmacol Ther 2008;83:234-42.

60. Crews KR, Gaedigk A, Dunnenberger HM, et al; Clinical Pharmacogenetics Implementation Consortium. Clinical Pharmacogenetics Implementation Consortium guidelines for cytochrome P450 2D6 genotype and codeine therapy: 2014 update. Clin Pharmacol Ther 2014;95:376-82.

61. Borges S, Desta Z, Jin Y, et al. Composite functional genetic and comedication CYP2D6 activity score in predicting tamoxifen drug exposure among breast cancer patients. J Clin Pharmacol 2010;50:450-8.

62. Hines RN. The ontogeny of drug metabolism enzymes and implications for adverse drug events. Pharmacol Ther 2008;118:250-67.

63. Furuta T, Iwaki T, Umemura K. Influences of different proton pump inhibitors on the anti-platelet function of clopidogrel in relation to CYP2C19 genotypes. Br J Clin Pharmacol 2010;70:383-92. 
64. Fernando H, Bassler N, Habersberger J, et al. Randomized double-blind placebo-controlled crossover study to determine the effects of esomeprazole on inhibition of platelet function by clopidogrel. J Thromb Haemost 2011;9:1582-9.

65. Gage BF, Eby C, Johnson JA, et al. Use of pharmacogenetic and clinical factors to predict the therapeutic dose of warfarin. Clin Pharmacol Ther 2008;84:326-31.

66. Klein TE, Altman RB, Eriksson N, et al; International Warfarin Pharmacogenetics Consortium. Estimation of the warfarin dose with clinical and pharmacogenetic data. N Engl J Med 2009;360:753-64.

67. Gardin A, Ufer M, Legangneux E, et al. Effect of Fluconazole Coadministration and CYP2C9 Genetic Polymorphism on Siponimod Pharmacokinetics in Healthy Subjects. Clin Pharmacokinet 2019;58:349-61.

68. Jin Y, Borell H, Gardin A, Ufer M, Huth F, Camenisch G. In vitro studies and in silico predictions of fluconazole and CYP2C9 genetic polymorphism impact on siponimod metabolism and pharmacokinetics. Eur J Clin Pharmacol 2018;74:455-64.

69. Relling MV, Schwab M, Whirl-Carrillo M, et al. Clinical Pharmacogenetics Implementation Consortium Guideline for Thiopurine Dosing Based on TPMT and NUDT15 Genotypes: 2018 Update. Clin Pharmacol Ther 2019;105:1095-105.

70. Yang JJ, Lim JY, Huang J, et al. The role of inherited TPMT and COMT genetic variation in cisplatin-induced ototoxicity in children with cancer. Clin Pharmacol Ther 2013;94:252-9.

71. Cheung ST, Allan RN. Mistaken identity: misclassification of TPMT phenotype following blood transfusion. Eur J Gastroenterol Hepatol 2003; $15: 1245-7$.

72. Kroon LA. Drug interactions with smoking. Am J Health Syst Pharm 2007;64:1917-21.

73. Kato H, Usui M, Muraki Y, et al. Intravenous Administration of Tacrolimus Stabilizes Control of Blood Concentration Regardless of CYP3A5 Polymorphism in Living Donor Liver Transplantation: Comparison of Intravenous Infusion and Oral Administration in Early Postoperative Period. Transplant Proc 2018;50:2684-9.

74. Fleming JN, Posadas Salas MA, Taber DJ. Dosing Requirements of Extended-Release Tacrolimus (Astagraf XL) in African American Kidney Transplant Recipients Converted from Immediate-Release Tacrolimus (AAAKTRS). Ther Drug Monit 2020;42:415-20.

75. Hines RN. Ontogeny of human hepatic cytochromes P450. J Biochem Mol Toxicol 2007;21:169-75.

76. Helldén A, Madadi P. Pregnancy and pharmacogenomics in the context of drug metabolism and response. Pharmacogenomics 2013;14:1779-91.

77. Betcher HK, George AL Jr. Pharmacogenomics in pregnancy. Semin Perinatol 2020;44:151222.

78. Koren G, Ornoy A. Clinical implications of selective serotonin reuptake inhibitors-selective serotonin norepinephrine reuptake inhibitors pharmacogenetics during pregnancy and lactation. Pharmacogenomics 2018;19:1139-45.

79. Bell GC, Caudle KE, Whirl-Carrillo M, et al. Clinical Pharmacogenetics Implementation Consortium (CPIC) guideline for CYP2D6 genotype and use of ondansetron and tropisetron. Clin Pharmacol Ther 2017;102:213-8.

80. Tracy TS, Venkataramanan R, Glover DD, Caritis SN; National Institute for Child Health and Human Development Network of MaternalFetal-Medicine Units. Temporal changes in drug metabolism (CYP1A2, CYP2D6 and CYP3A Activity) during pregnancy. Am J Obstet Gynecol 2005;192:633-9. 